\title{
Dependência morfométrica de carapaça e plastrão de Phrynops geoffroanus (SCHWEIGGER, 1812)
}

\section{Morphometric dependence of carapace and plastron of Phrynops geoffroanus (SCHWEIGGER, 1812)}

\author{
1 Joara de Sousa Andrade joaraandrade13@gmail.com \\ 1 Antonio Nilberto Grangeiro de Abreu Junior \\ 2 Diogo Brunno e Silva Barbosa \\ 3 Jonas Pederassi \\ ${ }^{4}$ Mauro Sérgio Cruz Souza Lima
}

\footnotetext{
1 Graduação em Ciências Biológicas pela Universidade Federal do Piauí.

2 Mestre, Universidade Federal do Piauí, Campus Amílcar Ferreira Sobral, Laboratório de Herpetologia.

3 Doutor, Associação Educacional Dom Bosco, AEDB.

4 Doutor, Universidade Federal do Piauí.
}

\section{Resumo}

Considerando a distribuição da espécie Phrynops geoffroanus (SCHWEIGGER, 1812), facilmente encontrada, foram capturados 20 espécimes com a utilização de armadilhas do tipo espera iscada que, posteriormente, foram depositadas na coleção de História Natural da Universidade do Piauí (CNHNUFPI 0007 - 0026). 0 presente trabalho objetivou estabelecer razões alométricas entre a maturidade e o crescimento do plastrão e da carapaça. As medidas foram submetidas ao coeficiente de Pearson, quando os valores foram superiores $(r=0,80)$. Submetemos as amostras à regressão univariada linear para o plastrão e exponencial para a carapaça, realizando 6 combinações possíveis. 0 valor de $r$ ajustado ao coeficiente de determinação, quando igual ou superior a 0,80 , foram considerados com correlação forte positiva. Dessa forma, apresentando variáveis dependentes, as análises morfométricas indicaram a existência de correlação inferior a 0,80 apenas nas combinações CLCxLLC ( $r=0,7191)$ e LLCxCP $(r=0,7364)$. Por meio da correlação de Pearson e o ajuste à equação linear e exponencial, é possível utilizar a função para prever medidas das placas, tamanho, proporção e a dependência de crescimento entre as porções da carapaça ou plastrão na população amostral de Phrynops geoffroanus. Tais predições foram confirmadas pelo teste de aderência X2 .

\section{Palavras-chave}

Alometria. Chelidae. Osteodermos. Razão de crescimento.

\begin{abstract}
Considering the distribution of the species Phrynops geoffroanus (SCHWEIGGER, 1812) which is easily found, 20 specimens were captured using bait-waiting traps, later deposited in the Natural History collection at the University of Piauí (CNHNUFPI 0007 - 0026). The present study aimed to establish allometric ratios between the maturity and growth of the plastron and carapace. The measures were submitted to Pearson's coefficient, when the values were higher than $r=0.80$, we submitted to linear univariate regression for the plastron and exponential for the carapace, making the six possible combinations. The value of $r$ adjusted to the coefficient of determination, when equal to or greater than 0.80 , we consider a strong positive correlation, thus presenting dependent variables. Morphometric analyzes indicated the existence of a correlation below 0.80 only in the combinations: linear carapace length $x$ linear carapace width $(r=0.72)$ and linear carapace width $x$ plastron length $(r=0.74)$. Using Pearson's correlation and adjusting the linear and exponential equation, it is possible to use the function to predict plate measurements, size, proportion and growth dependency between portions of the carapace or plastron in the sample population of Phrynops geoffroanus. These predictions were confirmed by the $X^{2}$ adherence test.
\end{abstract}

\section{Keywords}

Allometry. Chelidae. Osteoderms. Growth ratio.

\section{Como você deve citar?}

ANDRADE, Joara de Sousa et al. Dependência morfométrica de carapaça e plastrão de Phrynops geoffroanus (SCHWEIGGER, 1812). Cadernos UniFOA, Volta Redonda, n. 43, p. 143-150, agosto 2020. 


\section{INTRODUÇÃO}

Os Testudines são tetrápodes caracterizados por possuírem esqueleto dérmico que formam uma concha. A carapaça formada por ossos dérmicos e vértebras endocondrais correspondem à parte superior; a parte inferior fecha essa concha e é denominada plastrão (BURKE, 1991; CLARK et al., 2001; CARROLL, 2013). A concha das tartarugas auxilia na proteção física e mecânica, funcionando, em algumas espécies, como um reservatório de água ou gordura (GILBERT et al., 2001).

As tartarugas da subordem Pleurodira apresentam característica de retrair o pescoço lateralmente (KARDONG, 2016) e é composta por três famílias Chelidae, Pelomedusidae e Podocnemidae (BUJES, 2010). A Chelidae possui representantes conhecidos popularmente como cágados e, no Brasil, conta com 19 espécies que ocorrem em todo o país. Para o estado do Piauí, estão descritas três espécies: Mesoclemmys perplexa (BOUR; ZAHER, 2005) Mesoclemmys tuberculata (LUEDERWALDT, 1926) e Phrynops geoffroanus (SCHWEIGGER, 1812) (COSTA; BÉRNILS, 2018).

A espécie Phrynops geoffroanus geralmente é encontrada em rios, lagos ou córregos antropizados. Sua alimentação é basicamente carnívora: insetos aquáticos, girinos e pequenos peixes (MEDEM, 1966). Assim sendo, o estudo da morfometria dessa espécie é facilitado em razão da sua distribuição. A morfometria é um ramo da ciência que, a partir das medidas biométricas dos organismos, permite predizer resultados, estando associada à ontogenia que visa acompanhar o crescimento dos indivíduos e sua respectiva evolução filogenética (MANDARIM-DE-LACERDA, 1995).

Considerando a facilidade de amostragem de $P$. geoffroanus, iniciamos estudos morfométricos visando estabelecer razões alométricas entre a maturidade, crescimento do plastrão e carapaça, isto é, quando ocorre o crescimento corpóreo em razão da maturidade, também ocorre o crescimento proporcional da concha.

\section{MATERIAL E MÉTODOS}

\section{1 Área, Coleta e $\mathrm{N}$ amostral}

Os espécimes foram coletados no riacho do Leite, local antropizado, na cidade de Floriano, no estado do Piauí ( $\left.6^{\circ} 45^{\prime} \mathrm{S} 43^{\circ} 00^{\prime} \mathrm{W}\right)$. Os animais foram capturados com a utilização de armadilhas de espera iscada. $\mathrm{O} \mathrm{N}$ amostral foi de 20 espécimes que se encontram depositados na coleção de História Natural da Universidade do Piauí (CNHNUFPI 0007 - 0026). 0 estudo recebeu autorização de coleta e transporte Sisbio/IBAMA 61.118, pelo CEUA, Processo 23107.018093/2018-73 (UFAC).

\subsection{Morfometria}

Com o auxílio de paquímetro (precisão 0,01 mm), foram aferidos o comprimento linear da carapaça (CLC) da porção anteroposterior da região da placa cervical até a décima segunda placa marginal, alinhada pelas placas vertebrais e a largura linear da carapaça (LLC), medidas entre a $6^{\mathrm{a}} \mathrm{e} 7^{\mathrm{a}}$ marginais. 0 comprimento do plastrão (CP) foi aferido entre a porção anteroposterior da região da placa intergular à anal, estando o paquímetro alinhado à fissura medial, formada pela interseção das placas umerais, peitorais, abdominais, femorais e anais. A aferição da largura foi medida na fissura formada pela intercessão da placa abdominal com a peitoral, obtendo-se a largura do plastrão (LP). Os valores são apresentados em média \pm desvio padrão (intervalo de variação). 


\subsection{Análises morfométricas}

As aferições foram submetidas ao coeficiente de correlação de Pearson (r), cuja verificação da acuracidade do coeficiente de correlação foi submetida ao teste t de correlação de Student para avaliar se a amostragem foi suficiente. Quando os valores de correlação alcançaram os resultados superiores $(r=0,80)$, foram submetidos à regressão univariada linear para o plastrão e exponencial para a carapaça. Sua aderência foi testada pelo $X^{2}$ em n-1 graus de liberdade e valores de confiança de $95 \%$.

Através da análise combinatória: $C_{n, p}=n ! / p !(n-p) !$, onde $C$ representa a fórmula de combinação entre o comprimento e largura da carapaça e plastrão; n, quantidade de elementos do conjunto (CLC, CP, LLC e LP) e, p, a quantidade de elementos que irão formar as combinações, que, no presente estudo, correspondeu a 6 combinações possíveis (CLCxCP, LLCxLP, CLCxLLC, LPxCLC, LLCxCP, CPxLP).

\section{RESULTADOS E DISCUSSÃO}

Na morfometria de Phrynops geoffroanus, o CLC variou em 232,82 $( \pm 30,69)$, o LLC variou em $163,68( \pm 28,42)$, o CP variou em 183,84 $( \pm 26,16)$ e o LP variou em 131,16 $( \pm 17,60)$. Dentre os 20 espécimes analisados, apenas um jovem foi identificado, medindo 151,47 mm (carapaça) e 121,82 mm (plastrão). Os espécimes foram considerados maduros, quando o comprimento da carapaça foi igual ou superior a 197 mm (BOUR; ZAHER, 2005). Essa condição morfométrica de maturidade equivale à espécie silvestre em ambiente antropizado, diferindo $3 \mathrm{~mm}$ dos valores encontrados por Molina (1992) em cativeiro (Setor de Répteis da Fundação Parque Zoológico de São Paulo). Em seu trabalho, a medida mínima para os machos foi de $200 \mathrm{~mm}$ e, para as fêmeas, $235 \mathrm{~mm}$ de comprimento da carapaça.

O resultado da correlação de Pearson: $\operatorname{CLCxCP}(r=0,9663, t=15,9432)$ (Figura 1A), LLCxLP $(r=0,8083$, $t=5,8251$ ) (Figura 1B), CPxLP ( $r=0,9458, t=12,3658$ ) (Figura 1C) e CLCxLP $(r=0,9363, t=10,9663)$ (Figura 1D) demostra uma correlação forte positiva. Os testes indicaram a existência de correlação inferior a 0,80 apenas entre o comprimento da carapaça com a largura linear da carapaça CLCxLLC $(r=0,7191$, $t=4,3915)$ (Figura 1E) e largura linear da carapaça com o comprimento do plastrão LLCxCP $(r=0,7364$, $t=4,6183$ ) (Figura 1F). 
Figura 1 - Gráficos de correlação de Pearson do comprimento linear da carapaça (CLC), largura linear da carapaça (LLC), comprimento do plastrão (CP) e largura do plastrão (LP) na espécie Phrynops geoffroanus, medidas em $\mathrm{mm}$.
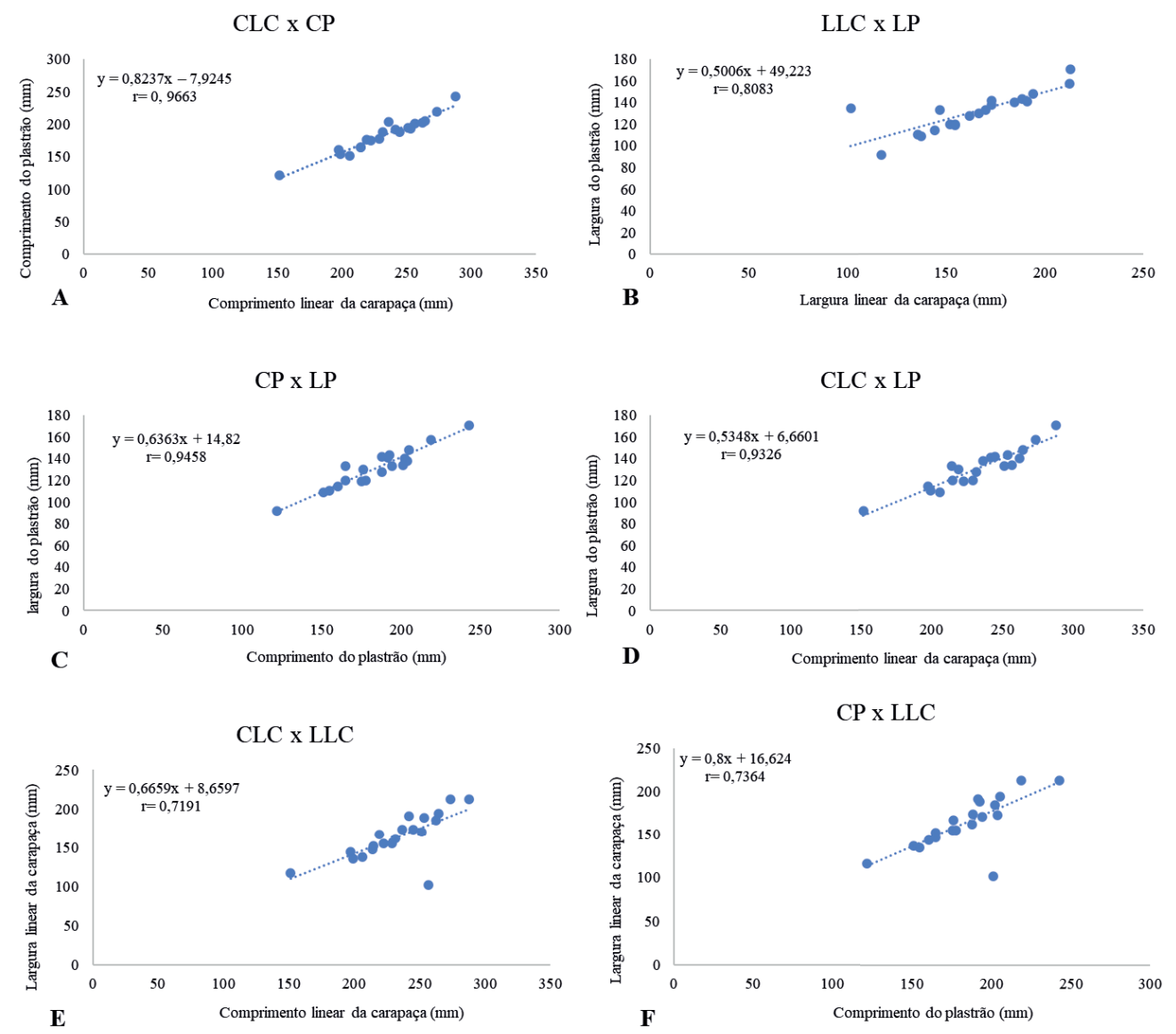

Fonte: Dos autores.

Nas combinações de CLCxLLC e CPxLLC, foram encontrados valores inferiores, sendo assim, não ocorreu correlação forte positiva. Rodrigues et al. (2005) encontraram valor da dependência maior na medida do $C L C$ em relação a LLC $(r=0,9748)$, porém o estudo foi relativo à espécie Podocnemis expansa (SCHWEIGGER, 1812), enquanto, em nosso estudo, para $P$. geoffroanus, o valor encontrado foi de $r=0,7191$.

Costa et al. (2017) estudaram a espécie Kinosternon scorpioides (LINNAEUS, 1766), observando correlação positiva entre as variáveis comprimento da carapaça e comprimento do plastrão $(r=0,7119)$ e entre o comprimento e a largura do plastrão $(r=0,5956)$. No entanto, em nosso trabalho, encontramos correlações positivas superiores (CLCxCP. $r=0,9663$ e CPxLP. $r=0,9458$ ). Só será possível inferir que tais diferenças são devido a distinções naturais entre espécies ou a variações ontogenéticas resultantes de condições ambientais distintas (fenotípicas) das populações comparadas, quando mais pesquisas nesse âmbito forem realizadas em diferentes populações de diferentes regiões e espécies do grupo.

Pignati \& Pezzuti (2012), avaliando a morfometria de Podocnemis unifilis (TROSCHEL, 1848) para cinco variáveis (comprimento retilíneo da carapaça, largura da carapaça, comprimento do plastrão, largura da cabeça e massa), encontraram dependência entre o par de variável CLC e massa corporal $(r \geq 0,9512)$, registrando independência para todas as outras variáveis. Diferentemente, em nosso trabalho, encontramos dependência morfométrica nas seis combinações de comprimento e largura da carapaça, e comprimento e largura do plastrão, de forma semelhante. Costa et al. (2015) encontraram 
correlações positivas em todas as variáveis para Kinosternon scorpioide, acrescidos de duas outras variáveis, massa corporal e altura $(\mathrm{cm})$.

Ao ajustarmos as variáveis morfométricas à regressão linear CLC x LLC $(\mathrm{y}=0,6659 \mathrm{x}+8,6597(\mathrm{r}=0,71)$ e CP x LLC ( $y=0,8 x+16,624$ ( $r=0,73)$, não consideramos os ajustes em razão dos valores do coeficiente de correlação serem inferiores a 0,80. Para as variáveis morfométricas acima desse valor (Tabela 1), obtivemos o ajuste das regressões lineares: CLC $x \operatorname{CP}(y=0,8237 x-7,9245(r=0,96), \operatorname{LLC} x \operatorname{LP}(y=0,5006 x+$ 49,223( $r=0,80), \operatorname{CP} x \operatorname{LP}(y=0,6363 x+14,182(r=0,94)$ e CLC x LP ( $y=0,5348 x+6,6601$ ( $r=0,93)$. Rodrigues et al. (2005) estabeleceram a correlação e regressão linear, porém não avaliaram a predição das variáveis morfométricas dependentes, não ficando clara a aplicabilidade desse cálculo para o entendimento da morfometria de quelônios.

Tabela 1 - Variáveis morfométricas com a correlação forte positiva entre as combinações

\begin{tabular}{llll}
\hline Variáveis Morfométricas & & $\mathrm{r}$ & $\mathrm{t}$ \\
\hline CLC & $\mathrm{CP}$ & 0,9663 & $15,9432 *$ \\
\hline LLC & LP & 0,8083 & $5,8251 *$ \\
\hline CP & LP & 0,9458 & $12,3658 *$ \\
\hline CLC & LP & 0,9363 & $10,9663 *$ \\
\hline \multicolumn{4}{c}{ * significativos }
\end{tabular}

Fonte: Dos autores.

Em nosso estudo, buscamos verificar aderência das equações das regressões lineares com a possibilidade de prever a variável dependente $(x ; y)$. Ao calcularmos a aderência ao nível de significância de $5 \%$, todos os resultados aderiram, confirmando a veracidade dos dados encontrados a partir da equação $(Y=a+b x)$, no valor tabelado ao nível de significância de 1\% (Tabela 2).

Tabela 2 - Variáveis morfométricas e respectivas equações de predição de crescimento. $(P=0,01)$

\begin{tabular}{lllll}
\hline Variável & $\mathrm{r}=$ & Equação & Valor de $x 2$ & Valor Tabelado a 99\% \\
\hline CLCxCP & 0,9663 & $\mathrm{y}=0,8237 x-7,9245 *$ & 4,827961 & \\
\hline LLCxLP & 0,8083 & $\mathrm{y}=0,5006 x+49,223^{*}$ & 19,5026 & \\
\hline CPxLP & 0,9458 & $y=0,6363 x+14,182 *$ & 5,04014 & 36,19 \\
\hline CLCxLP & 0,9326 & $y=0,5348 x+6,6601 *$ & 6,13223 & \\
\hline CCxLC & 0,7191 & $y=0,6659 x+8,6597 * *$ & 43,9171 & \\
\hline CPxLC & 0,7363 & $y=0,8 x+16,624 * *$ & 41,7975 & \\
\hline
\end{tabular}

* Diferenças signifıcativas / ** Não significativas

Fonte: Dos autores.

Quando a correlação de dependência foi superior a 0,80, obtivemos resultados satisfatório. Ao submetemos as variáveis morfométricas à equação da reta, os resultados apresentaram-se significativos, no entanto, quando os valores inferiores a estes não se ajustaram à equação, mostraram não serem significativos. Oktaviani et al. (2008) utilizaram, em seu trabalho, a equação linear nas espécies Amyda cartilaginea $(y=0,7523 x+2,5254)$ e Dogania subplana $(y=0,8217 x-1,2797)$, no entanto não realizaram as predições morfométricas, o que dificulta a compreensão das variáveis morfométricas. 
Quelônios formam uma concha, cuja região ventral é retilínea (plastrão), enquanto a região dorsal é curvilínea (carapaça) (SALERA-JUNIOR; MALVASIO; PORTELINHA, 2009). Assim sendo, não foi possível ajustar a carapaça com a regressão linear, no entanto, quando submetido à função exponencial (Figura 2), o ajuste da carapaça foi possível, com correlação de r=0,94, o teste de aderência calculado $\left(x^{2}=16,48\right)$, ao nível de significância de $1 \%$, quando comparado com o valor tabelado, foi menor, indicando que houve aderência.

Figura 2 - Curva de ajuste exponencial entre a proporção e razão das placas que compõem a carapaça

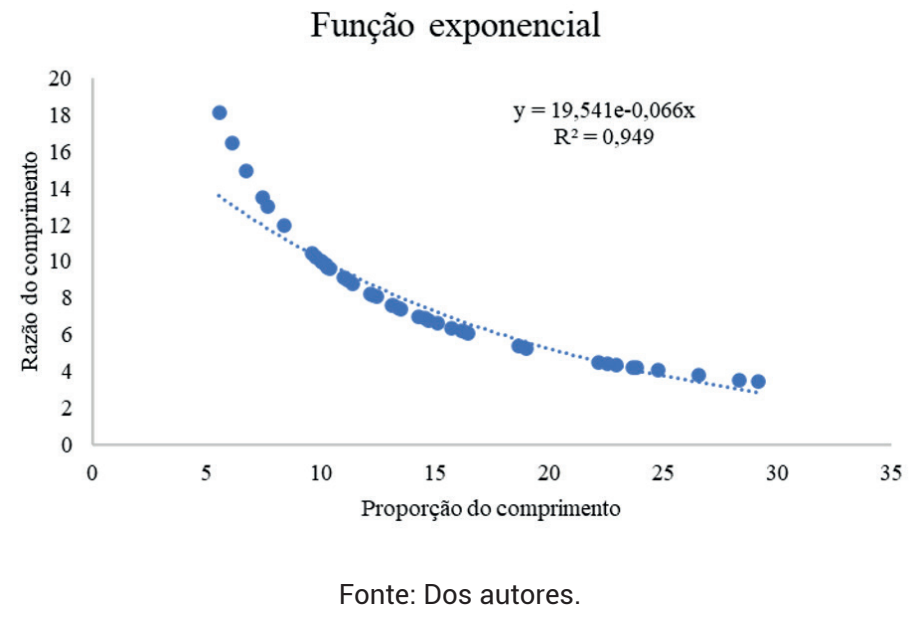

A relação entre duas variáveis e sua dependência morfométrica já foi fundamentada por Larcerda (1995) e Mather (1968), fontes determinísticas para a identificação da variável dependente explanatória. Diversos são os trabalhos que utilizaram análises dessas correlações através dos seus coeficientes estatísticos, como em Rodrigues et al. (2005) e Lima et al. (2012).

Alguns autores utilizaram a regressão exponencial para ajustar a morfometria dos indivíduos, como no trabalho de Flexa et al. (2005), em que ajustaram o peso e comprimento da espécie Macrobrachium amazonicum (Crustacea: Decapoda) ao modelo exponencial; Lima et al. (2012) realizaram a regressão exponencial nas variáveis corpóreas, avaliando o crescimento e alometria de Caiman latirostris (Chordata: Crocodylia); Gomiero et al. (2009) ajustaram o coeficiente de determinação, avaliando medidas morfométricas na espécie Brycon orbignyanus (Chordata: Characiformes); Botelho et al. (2019) analisaram a determinação da curva de crescimento e alometria nas espécies Piaractus mesopotamicus e Colossoma macropomum (Chordata: Characiformes), considerando o peso corporal e as medidas morfométricas em função da idade, apresentando um alto coeficiente de determinação.

\section{CONCLUSÃO}

Por meio da correlação de Pearson, foi possível utilizar a função para prever medidas das placas e a dependência de crescimento entre as porções da carapaça ou plastrão. Observou-se a existência de correlação entre o crescimento da carapaça em ajuste exponencial de crescimento, demostrando-se que é possível prever o tamanho e proporção do plastrão e carapaça para a população de Phrynops geoffroanus do Riacho do Leite, considerando o ajuste dos osteodermos. Dessa forma, ajustes lineares não permitem estabelecer correlações, sendo necessários ajustes exponenciais para obtenção da dependência de crescimento entre carapaça e plastrão. Cabe ressaltar ainda que a morfometria é de suma importância, uma vez que auxilia no estudo da morfologia e da taxonomia e assim contribui para compreensão da evolução, ontogenia e filogenia do grupo. 


\section{REFERÊNCIAS}

BOTELHO, H. A. Determinação da curva de crescimento e alometria de pacu (Piaractus mesopotamicus), tambaqui (Colossoma macropomum) e seus híbridos. Revista Agrarian, v. 12, p. 97-103, 2019.

BOUR, R.; ZAHER, H. A new species of mesoclemmys, from the open formations of northeastern Brazil (Chelonii, Chelidae). Papeis Avulsos de Zoologia, v. 45, n. 24, p. 295-311, 2005.

BUJES, C. S. Os Testudines continentais do Rio Grande do Sul , Brasil : taxonomia, história natural e conservação. Iheringia - Série Zoologia, v. 100, n. 4, p. 413-424, 2010.

BURKE, A. C. The Development and Evolution of the Turtle Body Plan : Inferring Intrinsic Aspects of the Evolutionary Process from Experimental Embryology. American Society of Zoologists, p. 616-627, 1991.

CLARK, K; BENDER, G; MURRAY, B. P; PANFILIO, K; COOK, S; DAVIS, R; MURNEN, K; TUAN, R. S; GILBERT, S. F. Evidence for the Neural Crest Origin of Turtle Plastron Bones. Genesis, p. 111-117, 2001.

CARROLL, R. L. Problems of turtle ancestry. in: brinkman d., holroyd p., gardner j. (eds) morphology and evolution of turtles. vertebrate paleobiology and paleoanthropology. Springer, dordrecht 2013.

COSTA, H.C.; BÉRNILS, R.S. Répteis do Brasil e suas Unidades Federativas: Lista de espécies. Revista de Herpetologia Brasileira, Piraquara-PR, v. 7, n. 1, p. 11-42, 2018.

COSTA, J. DA S; FIGUEIRÓ, M. R; MARQUES, L. S; SCHIERHOLT, A. S; MARQUES, J. R. F. Comportamento produtivo de muçuãs (Kinosternon scorpioides spp. Linnaeus, 1766). Ilha de Marajó, PA. Embrapa Amazônia Oriental, p. 1-4, 2015.

COSTA, J. S; MARQUES, L.C; SILVA, C. S; FIGUEIRÓ, M. R; SALES, R.L; DA SILVA FILHO, E; GUIMARÃES, D. A. A; MARQUES, J. R. F. Características produtivas de Kinosternon scorpioides nas fases de acasalamento, postura e eclosão, criados em cativeiro na Amazônia. Archivos de Zootecnia, p. 387-394, 2017.

FLEXA, C. E.; SILVA, K. C. A.; CINTRA, I. H. A. MORFOMETRIA DO CAMARÃO-CANELA, Macrobrachium amazonicum(HELLER, 1862), NO MUNICÍPIO DE CAMETÁ - PARÁ. Boletim Técnico Científico do CEPNOR, v. 5, n. 1, p. $41-54,2005$.

GILBERT, S. F; LOREDO, G. A; BRUKMAN, A; BURKE, A.C. Morphogenesis of the turtle shell: The development of a novel structure in tetrapod evolution. Evolution and Development, v. 3, p. 47-58, 2001.

GOMIERO, J. S. G. et al. CURVAS DE CRESCIMENTO MORFOMÉTRICO DE PIRACANJUBA (Brycon orbignyanus). Ciência e Agrotecnologia, v. 33, n. 3, p. 882-889, 2009.

KARDONG, KENNETH V. Vertebrados: anatomia comparada, função e evolução. 7. ed. Rio de Janeiro: Guanabara Koogan, 2016.

LIMA, M. S. C. S; PEDERASSI, J.; SOUZA, C. A. S. Avaliação do crescimento e alometria de Caiman latirostris (Crocodylia, Alligatoridae) em condições seminaturais de manejo. Cadernos UniFOA, v. 19, p. 73-80, 2012.

MANDARIM-DE-LACERDA, C. A. Métodos quantitativos em morfologia. Rio de Janeiro: EdUERJ, 1995. 
MATHER, K. Elementos de biometria. Tradução de Álvaro Marchi e outros. São Paulo: Polígon e Editôra da Universidade de São Paulo, 1968.

MEDEM, F. Contribuiciones al conocimiento sobre la ecologia y distribucion geografica de Phrynops (BATRACHEMYS) Dahli; (TESTUDINATA, PLEURODIRA, CHELIDAE). Caldasia, v. 9, p. 467-489, 1966.

MOLINA, F. DE B. Observações sobre o comportamento agnístico de cágados Phrynops geoffroanus (Schweigger, 1812) (Reptilia, Testudines, Chelidae) em cativeiro. Biotemas, p. 79-84, 1992.

OKTAVIANI, D; ANDAYANI, N; KUSRINI, M. D; NUGROHO, D. Identifikasi Dan Distribusi Jenis Labi-Labi. J. Lit. Perikan., v. 14, p. 145-157, 2008.

PIGNATI, M. T.; PEZZUTI, J. C. B. Alometria reprodutiva de Podocnemis unifilis (Testudines: Podocnemididae) na várzea do baixo rio Amazonas, Santarém, Pará, Brasil. Iheringia - Serie Zoologia, v. 102, n. 1, p. 48-55, 2012.

RODRIGUES, M. DE J. J; CARDOSO, E. DA C.; CINTRA, I. H. A.; SOUZA, R. F. C. Morfometria e redimento de carapaça de tartaruga-da-Amazônia, Podocnemis expansa (SCHWEIGGER, 1812) em ambiente natural. Revista Ciências Agrárias, p. 161-168, 2005.

SALERA-JUNIOR, G.; MALVASIO, A.; PORTELINHA, T. C. G. Avaliação de padrão irregular dos escudos do casco em Podocnemis expansa e Podocnemis unifilis (Testudines, Podocnemididae). Acta Amazônica, v. 39, p. $429-436,2009$. 